\title{
Cloud Armor: A Conclusion Work on Trust Management System
}

\author{
Lakshmi G S ${ }^{1}$, Shashirekha $\mathrm{H}^{2}$ \\ ${ }^{1}$ MTech student Department of CSE, VTU PG Center, Mysore, India \\ ${ }^{2}$ Assistant Professor Department of Computer science \&engineering, VTU PG center, Mysore, India
}

\begin{abstract}
Distributed computing gives Software as service (Saas) ,Platform as service(Paas) and Infrasrtucture as a service(Iaas).Cloud figuring condition having a few issues like protection and security. Trust administration is a standout amongst the most difficult issue. Shielding a specific cloud benefit from a few assaults like agreement attack(Such as client may give misdirecting criticism about specific cloud services)and Sybil attack(such as a solitary client can make numerous accounts..i.,e noxious user).In this paper we talk about cloudarmor that gives notoriety based trust administration to cloud administrations. A notoriety based trust administration system gives set of functionalities to convey Trust as a service(Taas) . Taas incorporates i)Zeroknowledge believability evidence convention to demonstrate validity of the consumers criticism and jam client security, ii)a validity show it will measures te validity of input to shield cloud administrations from malevolent client. what's more, iii)an accessibility model to deal with the accessibility of the trust administration.
\end{abstract}

Keyword-Cloud computing, Trust administration ,cloud amror, Reputation, credibility.

\section{INTRODUCTION}

The exceptionally unique, disseminated, anonstraightforward nature of cloud administrations make the trust administration in cloud conditions a noteworthy test [1], [2], [3], [4]. As indicated by specialists at Berkeley [5], trust and security are positioned one of the main 10 deterrents for the selection of cloud computing. Cloud figuring gives cost productive chances to undertakings by offering an assortment of dynamic, adaptable, and shared administrations. For the most part, cloud suppliers give confirmations by determining specialized and practical depictions in Service Level Agreements (SLAs) for the administrations they offer. The depictions in SLAs are not steady among the cloud suppliers despite the fact that they offer administrations with comparative usefulness. Along these lines, clients don't know whether they can recognize a reliable cloud supplier just in view of its SLA.

To bolster the clients in dependably distinguishing reliable cloud suppliers, a multi-faceted Trust Management (TM) framework design for a distributed computing marketplace[6] This framework gives intends to recognize the dependable cloud suppliers as far as various attribute(e.g., security, execution, consistence) surveyed by different sources and foundations of trust data Shoppers criticism is the great source to asses the trust value of cloud administrations [7], But in all actuality the cloud administrations are encounters the pernicious practices like intrigue assault or Sybil assault from their users[6],[8].

We recognize the accompanying top snags of the trust administration in cloud condition

- Consumer security - The selection of distributed computing raise protection concerns[10].there si a dynamic cooperation happens between cloud shoppers and cloud specialist co-ops, the dynamic collaborations which may includes touchy data like name ,address, telephone numbers, email id's and behavioral data like with whom the buyer associated, what sort of cloud administration what not. There are a few instances of protection braches, for example, spillage of touchy data and behavioral information.[11] .

- Cloud benefit security - Cloud administrations encounters assaults from its clients.Attackers can drawback a cloud benefit by giving different misdirecting criticisms (i.e., plot assaults) or by making a few records (i.e., Sybil assaults). In reality, the location of such malevolent practices represents a few difficulties .It is exceptionally hard to recognize Sybil attacks[12]. At last, it is extremely troublesome anticipate when the noxious practices occurs[13].

- Trust administration benefit - A trust administration benefit gives an interface amongst clients and cloud administrations for powerful trust administration .Trust administration is troublesome because of capricious number of clients and the dynamic way of distributed computing environment [7].

In this paper we examine a Reputation Based Trust administration for cloud administrations i.e. Cloudarmor. In cloudarmor trust is conveyed as a service (Taas).cloudarmor misuses systems to distinguish trustworthy criticisms from pernicious clients.

Cloudarmor comprises of three elements

1. Zero-information believability verification protocol(ZKC2P)- ZKC2P is a novel convention that 
jam the customer security and it likewise empowers trust administration service(TMS) to demonstrate the validity of specific shoppers input .In this character administration (IdM) is utilized that helps TMS to gauge the validity of buyers criticism without breaking the buyers protection. IdM can encourage TMS in recognition of Sybil assaults against cloud administrations without breaking the protection of clients .When client endeavors to utilize TMS first time, TMS obliges them to enlist their certifications at the trust character registry in IdM .after that the IdM set up their personalities. The trust personality registry store every client credentials.TMS initiates the clients in light of their history records.

2. A validity demonstrate The believability of the buyers input is critical in TMS .In this few measurements are utilized to recognize assaults ,for the agreement assault criticism thickness and intermittent input plot. These measurements recognize misdirecting criticism from pernicious clients. It additionally can identify intrigue attack (I.e., aggressors who are expected to control the trust comes about by giving various inputs to specific cloud administrations). For Sybil assault multipersonality acknowledgment and intermittent Sybil assaults. These measurements enable TMS to recognize deceiving inputs from Sybil attack (i.e., attackers who are proposed to make various records to give deluding criticism for specific cloud administrations).

3. An accessibility show High accessibility is an essential prerequisite to the trust administration benefit. Along these lines an accessibility show proposed to spread a few disseminated hubs to deal with the criticisms given by clients in a decentralized way Load adjusting methods are misused to share the workload thereby continually keeping up a coveted accessibility level.

The rest of the paper is sorted out as follows section II section presents related work it comprises of related work done over trust and notoriety.segment III quickly exhibits the cloudarmor system and its characteristics. IV presents points of interest and weaknesses it incorporates burdens of existing framework and focal points of cloudarmor. finally last segment gives conclusion.

\section{RELATED WORK}

In the course of recent years, trust administration theme in the region of distributed computing [8], [13], [16]. A portion of the exploration endeavors utilize approach based trust administration procedures. For example,[17] propose Trust Cloud system for responsibility and trust in distributed computing. Specifically, Trust Cloud comprises of five layers including work process, information, framework, arrangements and laws, and directions layers to address responsibility in the cloud condition. These layers keep up the cloud responsibility life cycle which comprises of seven stages including strategy arranging, sense and follow, logging, safe-keeping of logs, detailing and replaying, inspecting, and enhancing and redressing. Brandic [7] propose a novel approach for consistence administration in cloud conditions to build up trust between various gatherings. The approach is produced utilizing a unified design and uses consistent administration system to build up trust between cloud benefit clients and cloud specialist co-ops.

Not at all like past works that utilization arrangement based trust administration methods, we evaluate the reliability of a cloud benefit utilizing notoriety based trust administration systems. Notoriety speaks to a high impact that cloud benefit clients have over the trust administration framework [18], particularly that the feelings of the different cloud benefit clients can significantly impact the notoriety of a cloud benefit either emphatically or adversely. Some examination endeavors likewise consider the notoriety based trust administration procedures.

As per Hatman: Intra-Cloud Trust Management for Hadoop - S. M. Khan and K. W. Hamlen, the creators cited on Data and calculation honesty and security are real worries for clients of distributed computing offices. Numerous generation level mists hopefully expect that all cloud hubs are similarly reliable when dispatching occupations; employments are dispatched in light of hub load, not notoriety. This expands their defenselessness to assault, since trading off even one hub suffices to degenerate the uprightness of many dispersed calculations. As indicated by Privacy, Security and Trust in Cloud Computing - S. Pearson, the creators cited on, Cloud figuring alludes to the basic framework for a developing model of administration arrangement that has the benefit of lessening expense by sharing processing and capacity assets, consolidated with an on-request provisioning system depending on a compensation for every utilization plan of action. These new components directly affect data innovation (IT) planning additionally influence customary security, trust and protection systems.

Trust is a basic part of distributed computing. We analyzed and ordered existing exploration and routine with regards to trust components for distributed computing in five categories- notoriety based, SLA confirmation based, straightforwardness instruments (self-appraisal and data uncovering), trust as an administration, and formal accreditation, review, and guidelines. Most present work on trust in the cloud concentrate barely on specific parts of trust; our proposition is this is lacking. Trust is a mind 
boggling social marvel, and a systemic perspective of trust instrument investigation is fundamental.

Much past research has been done on notoriety administration frameworks in applications extending from online sell-offs to Web benefit determination to distributed systems. eBay is one of the best known cases of a notoriety administration framework for an online closeout website.

\section{CLOUDARMOR FRAMEWORK}

The cloud covering structure depends on the Service Oriented Architecture(SOA),SOA conveys trust as a service.SOA and web administrations are a standout amongst the most essential empowering advancements for distributed computing as in assets (framework ,stage and programming) are uncovered in cloud as services[14][15]. The beneath figure 1 describes the cloudarmor structure. which comprises of three layers specifically the cloud specialist organization layer, the trust administration benefit layer and the cloud customer layer. Three layers having their own particular cooperation with each of the layers and furthermore they are communicating with personality administration service(IdM).It contains character registry.

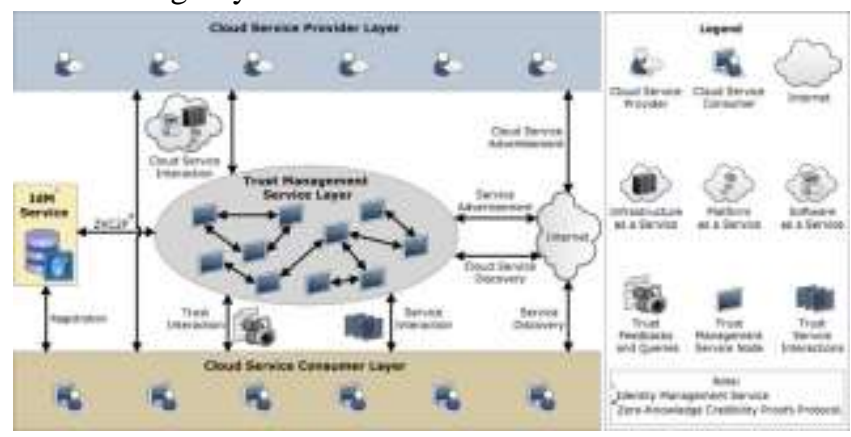

Fig.1:cloudarmor Architecture

The cloud specialist organization layer.- This layer comprises of various cloud specialist organizations who offer one or a few cloud administrations, i.e., Infrastructure as a Service (IaaS), Platform as a Service (PaaS), and Software as a Service (SaaS), freely on the web .These cloud administrations are available through web-based interfaces and recorded on web crawlers, for example, Google, Yahoo, and Baidu. Communications for this layer incorporate i) cloud benefit association with clients and TMS, and ii) cloud administrations notices where suppliers can promote their administrations on the web.

The trust administration benefit layer-This layer comprises of a few dispersed TMS hubs which are facilitated in numerous cloud conditions in various land ranges. These TMS hubs uncover interfaces so clients can give their criticism or the trust brings about a decentralized way. Associations for this layer include: i) cloud benefit cooperation with cloud specialist co-ops, ii) benefit notice to promote the trust as an administration to clients through the Internet, iii) cloud benefit disclosure through the Internet to enable clients to survey the trust of new cloud administrations, and iv) Zeroknowledge believability verification convention connections empowering TMS to demonstrate the validity of a specific shopper's input.

The cloud benefit shopper layer-Finally, this layer comprises of various clients who utilize cloud administrations. For instance, another startup that has restricted financing can devour cloud administrations (e.g., facilitating their administrations in Amazon S3). Connections for this layer include: i) benefit revelation where clients can find new cloud administrations and different administrations through the Internet, ii) trust and administration associations where clients can give their input or recover the trust aftereffects of a specific cloud administration, and iii) enrollment where clients set up their character through enlisting their certifications in IdM before utilizing TMS.

This structure additionally misuses a web slithering methodology for programmed cloud administrations revelation, where cloud administrations are consequently found on the Internet and put away in a cloud administrations vault. Besides, our structure contains a personality administration benefit (see Figure. 1) which is in charge of the enrollment where clients enlist their qualifications before utilizing TMS and demonstrating the believability of a specific shopper's criticism through ZKC2P.

\section{ADVANTAGES AND DISADVANTAGES}

Impediments of existing framework

- Guaranteeing the accessibility of TMS is a troublesome issue because of the capricious number of clients and the exceptionally unique nature of the cloud condition.

- A Self-advancing assault may have been performed on cloud benefit sy, which implies sx ought to have been chosen.

- Disadvantage a cloud benefit by giving various deluding trust inputs (i.e., agreement assaults)

- Trick clients into trusting cloud benefits that are not dependable by making a few records and giving deluding trust inputs (i.e., Sybil assaults).

\section{Points of interest of cloudarmor}

- TrustCloud system for responsibility and trust in distributed computing. Specifically, TrustCloud comprises of three layers including work process.

- Propose a multi-faceted Trust Management (TM) framework design for distributed computing to help the cloud benefit clients to recognize reliable cloud specialist organizations. 
- Zeroknowlegde validity evidence convention utilize IdM set up the credentilas of usersand TMS initiates the tust client.

\section{CONCLUSION}

Cloud benefit clients criticism is the great source to asses the general reliability of cloud administrations .However vindictive clients may team up to inconvenience a cloud benefit by giving different deluding input and the clients make numerous records. Here in this paper we examine about the structure that gives set of functionalities to convey trust as an administration. Cloudarmor is a supporting notoriety based trust administration for cloud services.it includes novel methods that aides in distinguishing notoriety based assaults and enable client to recognize the reliable cloud service.A ccredible model that recognizes the deceptive criticisms from plot and Sybil assaults and an accessibility model that keeps up the trust administration benefit at the coveted level.

The future work is to consolidate diverse trust administration methods, for example, notoriety and suggestion to expand the trust result accuracyand execution streamlining of the trust administration administrations is another future work.

\section{ACKNOWLEDGEMENTS}

A sincere thanks to Mrs Shashshirekha $\mathrm{H}$ Assistant Professor Department of CS\&E VTU PG center Mysore. Dr K thippeswamy Head of the department CS\&E Vtu PG center Mysore And to my friends.

\section{REFERENCES}

[1] S. M. Khan and K. W. Hamlen, "Hatman: Intra-cloud trust management for Hadoop," in Proc. 5th Int. Conf. Cloud Comput., 2012, pp. 494-501.

[2] S. Pearson, "Privacy, security and trust in cloud computing," in Privacy and Security for Cloud Computing, ser. Computer Communications and Networks. New York, NY, USA: Springer, 2013, pp. 3-42.

[3] J. Huang and D. M. Nicol, "Trust mechanisms for cloud computing," J. Cloud Comput., vol. 2, no. 1, pp. 1-14, 2013.

[4] K. Hwang and D. Li, "Trusted cloud computing with secure resources and data coloring," IEEE Internet Comput., vol. 14, no. 5, pp. 14-22, Sep./Oct. 2010.

[5] M. Armbrust, A. Fox, R. Griffith, A. Joseph, R. Katz, A. Konwinski, G. Lee, D. Patterson, A. Rabkin, I. Stoica, and M. Zaharia, "A view of cloud computing," Commun. ACM, vol. 53, no. 4, pp. 5058, 2010.

[6] S. Habib, S. Ries, and M. Muhlhauser, "Towards a trust management system for cloud computing," in
Proc. 10th Int. Conf. Trust, Security Privacy Comput. Commun., 2011, pp. 933-939.

[7] I. Brandic, S. Dustdar, T. Anstett, D. Schumm, F. Leymann, and R. Konrad, "Compliant cloud computing (C3): Architecture and language support for user-driven compliance management in clouds," in Proc. 3rd Int. Conf. Cloud Comput., 2010, pp. 244-251.

[8] T. H. Noor, Q. Z. Sheng, S. Zeadally, and J. Yu, "Trust management of services in cloud environments: Obstacles and solutions," ACM Comput. Surv., vol. 46, no. 1, pp. 12:1-12:30, 2013.

[9] T. H. Noor, Q. Z. Sheng, and A. Alfazi, "Reputation attacks detection for effective trust assessment of cloud services," in Proc. 12th Int. Conf. Trust, Security Privacy Comput. Commun., 2013, pp. 469476.

[10] S. Pearson and A. Benameur, "Privacy, security and trust issues arising from cloud computing," in Proc. 2nd Int. Conf. Cloud Comput., 2010, pp. 693-702.

[11] S. Pearson and A. Benameur, "Privacy, security and trust issues arising from cloud computing," in Proc. 2nd Int. Conf. Cloud Comput., 2010, pp. 693-702.

[12]E. Friedman, P. Resnick, and R. Sami, "Manipulation-resistant reputation systems," in Algorithmic Game Theory. New York, USA: Cambridge Univ. Press, 2007, pp. 677-697.

[13] K. Ren, C. Wang, and Q. Wang, "Security challenges for the public cloud," IEEE Internet Comput., vol. 16, no. 1, pp. 69-73, Jan./Feb. 2012.

[14] T. Dillon, C. Wu, and E. Chang, "Cloud computing: Issues and challenges," in Proc. IEEE 24th Int. Conf. Adv. Inf. Netw. Appl., 2010, pp. 27-33.

[15] Y. Wei and M. B. Blake, "Service-oriented computing and cloud computing: Challenges and opportunities," IEEE Internet Comput., vol. 14, no. 6, pp. 72-75, Nov./Dec. 2010.

[16] K. Hoffman, D. Zage, and C. Nita-Rotaru, “A survey of attack and defense techniques for reputation systems," ACM Comput. Surv.,vol. 42, no. 1, pp. 131, 2009.

[17] R. Ko, P. Jagadpramana, M. Mowbray, S. Pearson, M. Kirchberg, L. Qianhui, and L. B. Sung, "TrustCloud: A framework for accountability and trust in cloud computing," in Proc. IEEE World Congr. Services, 2011, pp. 584-588.

[18] C. Dellarocas, "The digitization of word of mouth: Promise and challenges of online feedback mechanisms," Manage. Sci., vol. 49 no. 10, pp. 1407-1424, 2003. 